\title{
Tolerance of low-digestible carbohydrates: a general view
}

\author{
Geoffrey Livesey* \\ Independent Nutrition Logic, Pealerswell House, Bellrope Lane, Wymondham, Norfolk, NR18 OQX, UK
}

\begin{abstract}
The tolerance of low-digestible carbohydrates (LDCs) may be measured as the potential to cause abdominal symptoms and laxation. Tolerance of any one LDC is determined by its concentration in the food product eaten, the amount of the food product eaten, the frequency of eating the food and the consumption of other foods (increasing tolerance) and water (decreasing tolerance). Added to these, individuals vary considerably in their response to low-digestible carbohydrates in the reporting of gastrointestinal symptoms. A precise maximum no-response dose is sometimes difficult to obtain because some dose-response curves are distinctly sigmoidal. Food regulators hoping to set a trigger level at which laxation may occur have been unable to take account of all these factors because the necessary information matrices are not available for any one LDC. Nevertheless analysis of the data shows consistent trends and for circumstances when food is consumed throughout the day it now seems feasible to assign specific tolerances to specific low-digestible carbohydrates, especially the polyols for which most is known. The method by which the no-effect dose or laxative threshold is expressed is critical to its application to individual foods.
\end{abstract}

Dietary fibre: Polyols: Laxation: Nausea: Emesis: Humans: Food regulations

Low-digestible carbohydrates (LDCs) present in or intended for human foodstuffs (Table 1) offer both potential benefits and some risks (Table 2). Consumer acceptance of LDCs is inevitably a subjective balance of perceived risks and benefits, some of which extend beyond those tabulated and lead to personal preferences. More objective criteria are used to assess physiological tolerance of LDCs. Such tolerance is monitored as adverse effects, or intolerance; for example the occurrence of laxation. The quantity of quality information and apparent multiplicity of factors affecting tolerance to LDCs precludes more than semiquantitative predictions about the extent to which consumers respond to them physiologically and how food regulators might use the available information to advise the consumer. The present review aims to put the subject into context and should be considered neither comprehensive nor definitive. Issues are raised but final conclusions are not sought, although it is intended to bring some clarity to the subject.

\section{Potential benefits}

Early considerations were for unrefined carbohydrates, such as those comprising dietary fibre. These have been considered to be a marker of a healthy type of diet (British Nutrition Foundation, 1990). Other early considerations were for refined carbohydrates, such as sorbitol (hydrogenated glucose). Like sucrose, sorbitol is sweet but unlike sucrose contributes little to the plasma glucose and insulin responses. Hydrogenated carbohydrates (sugar alcohols or polyols), from sorbitol to the most recent introduction of erythritol, have been considered helpful therefore to maturity onset (now type II) diabetics (Ellis \& Krantz, 1941; Bornet et al. 1996). This trend is continuing. Now highly digestible starches are considered possibly more damaging to health than sucrose, which is less glycaemic. The majority of starch in foodstuffs generate glucose rapidly and so promote substantial glycaemia (Miller \& Lobbezoo, 1994). Low-digestible starches probably offer benefits (or less risk) in this regard along with other lowdigestible carbohydrates (Jenkins et al. 1981). Other considerations are for tooth friendliness (Kandelman, 1997; Moynihan, 1998), low energy value (Livesey, 1992), value as laxatives (Lederle et al. 1990; Tramonte et al. 1997), prebiotic value as modifiers of colonic ecology and protection against enteric pathogens (Collins \& Gibson, 1999; Cummings \& MacFarlane, 1999), faecal bulking characteristics potentially helpful in prevention of cancer of the colon (Cummings et al. 1992), and plasma lipid modification in favour of reduced coronary heart disease (Jenkins \& Kendall, 1998). Other benefits, such as adding bulk to the diet as an appetite suppressant is of uncertain value in the long-term in normal adults, but it may be important to avoid large amounts in children due to their higher energy requirements, especially when digestion is compromised for other reasons (Gavin et al. 1997), although even this risk may now be offset by so much television watching. In addition, the products of LDC

\footnotetext{
* Corresponding author: G. Livesey, fax + 44 (0)1953 600218, email glivesey@inlogic.co.uk
} 
Table 1. Low-digestible carbohydrates in the human diet

\begin{tabular}{l}
\hline Dietary fibre polysaccharides \\
Pectins \\
Ispahula \\
Fibogel \\
Celluloses \\
Inulin \\
Resistant starches \\
Various forms \\
Oligosaccharides \\
Polydextrose \\
Fructo-olgosaccharides \\
Sugar alcohols \\
Lactitol \\
Isomalt \\
Maltitol \\
Sorbitol \\
Mannitol \\
Xylitol \\
Erythritol \\
Rare sugars \\
L-Sugars \\
D-Tagatose \\
Lactulose \\
Trehalose \\
Isomaltulose \\
\hline
\end{tabular}

fermentation may be essential for the health of the colon as reviewed elsewhere (see Scheppach et al., this issue; Livesey \& Elia, 1996).

There are, therefore, many potential benefits from consuming low-digestible carbohydrates. The extent and nature of these benefits depend on the presentation and nature of the LDCs. The benefits of LDCs are maintained or enhanced when they are present in fat-free or reduced-fat products, consumed in moderation, packaged so as not to be confused with high-glycaemic foods (such as used to correct hypoglycaemia in diabetics) and when consumed in accordance with local dietary guidelines. No LDCs are without one or another physiological effect. Some LDCs are strongly functional, particularly in the laxation they cause and the dental caries they help prevent. Limiting their usefulness are the adverse physiological responses they evoke, in particular those with a low molecular weight, which may cause laxation. To limit such adverse responses and to advise consumers of the causes, food regulators world-wide have generated various advisory statements for the purpose of food labelling (Zumbé et al., this issue; Howlett, this issue). So far as possible, it has become important to quantify the potential risks or adverse responses, in particular the potential for laxation in 'normal' people.

\section{Potential risks}

The benefits that LDCs bring are limited by their tolerance - all increase the rate of fermentation in the colon, causing abdominal discomfort, flatus and potentially diarrhoea, the latter especially when the LDC is of low molecular weight. Circumstances that lead to least discomfort from LDCs are of interest because these release a potential to benefit from them as part of a healthy diet. The risk of laxation and abdominal discomfort may be alarming and lead to inappropriate medical intervention when the cause has gone unrecognised (Canada, 1993); it is the intent that food regulations avoid such occurrence. However, symptoms of excess LDC ingestion are far from life threatening and so require no prescriptive warnings of their effects (cf. tobacco warnings), just advice about the cause. Laxation more than abdominal discomfort appears to be of concern since advisory statements apply to low rather than highmolecular-weight LDCs such as those present in dietary fibre.

\section{Perceptions of risk and benefit: equality for LDCs?}

From a scientific and trade perspective, it is desirable to examine all the important LDCs equally for their potential benefits and adverse responses. The important benefits offered by LDCs (Table 2) are largely impossible to perceive by individuals, although they may be learned. This is because the majority of benefits are accrued in the long term, may be perceived as modest in extent because they are difficult to prove confidently, and are observable in the main as statistical phenomena in predetermined measurements on large numbers of people. In addition, data may not be available for particular LDC products; rather benefits inferred may be based on a response to LDCs

Table 2. Potential risks and benefits of low-digestible carbohydrates*

\begin{tabular}{|c|c|}
\hline Potential benefits & $\begin{array}{l}\text { Low plasma glucose and insulin excursions (care of type II diabetes and possibly colon cancer) } \\
\text { Plasma lipid modification (coronary heart disease) } \\
\text { Low oral acidification (dental caries prevention) } \\
\text { Low energy value (obesity aid) } \\
\text { Value as laxatives (constipation) } \\
\text { Prebiotic value (tolerance/control of enteric pathogens) } \\
\text { Dietary bulking (satiating, obesity) } \\
\text { Stool bulking/dilution (colon cancer, irritable bowl) } \\
\text { Generation of butyrate and acidification of the colon (health of the colon) }\end{array}$ \\
\hline Potential risks & 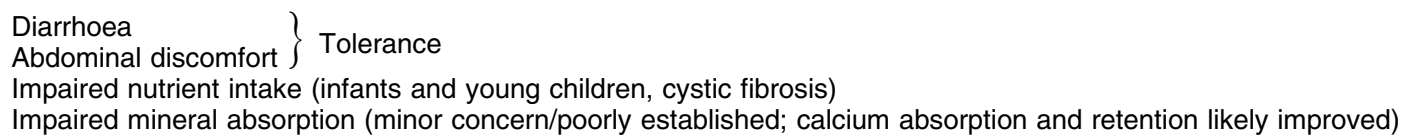 \\
\hline
\end{tabular}

* The benefits and risks are not universal to all low-digestible carbohydrates (see text). 
Table 3. Response terms used when assessing tolerance of LDCs; those that are not symptomatic of excess LDC consumption are given in italics

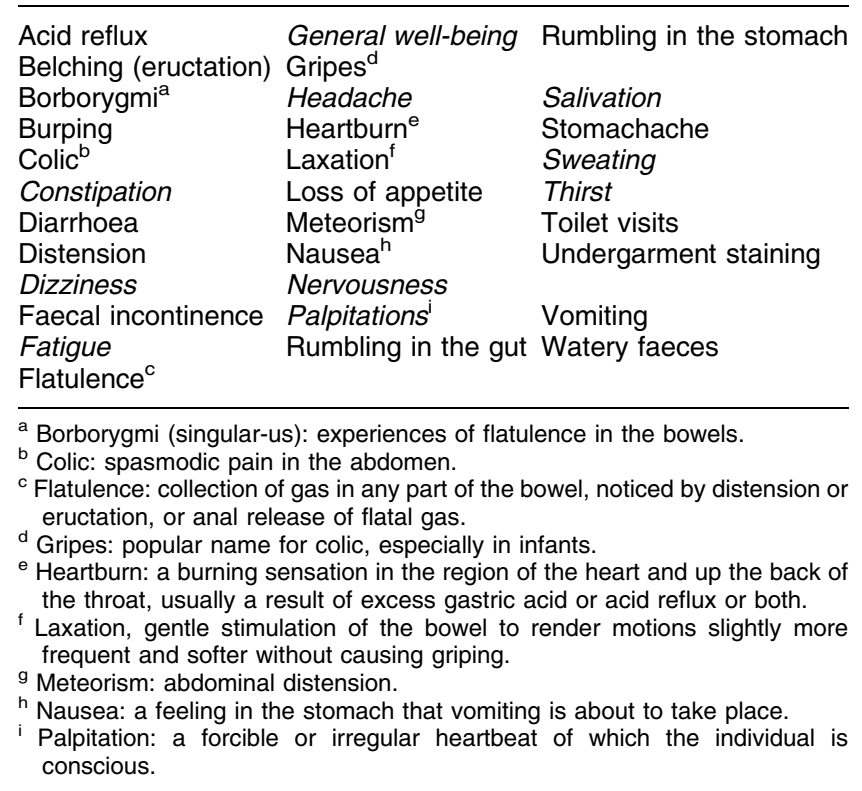

generically. An exception is laxation, which when experienced brings immediate constitutional relief. Although dietary bulk contributes to feelings of fullness and faecal bulking to the frequency of toilet visits, the benefits from these (Table 2) are understood through education rather than perceived directly. By contrast, individuals readily perceive those responses to LDCs that are implicit risks and which are investigated usually under the headings of adverse effects or tolerance. Such responses (Table 3) impact seemingly with spontaneity, are almost immediate, and can be evidenced by the individual.

Because the benefits of LDCs are not readily perceived, they need to be teased out and explained, for example in dietary guidelines. The risks being readily experienced by individuals are treated differently, with advisory statements on food labels. This has generated an important issue. The collective guidance and labelling advice does not treat all LDCs equally. Dietary guidelines tend to promote health through positive messages and so tend to promote dietary fibre. Food labelling advice focuses on the risks and so promotes a negative image, e.g. of laxative carbohydrates. De facto, description of risks and benefits in different places has the potential to confuse the consumer about the value of a certain foodstuff.

Not all risks appear to be treated equally either. The presence of lactose as a causative factor of symptoms of lactose intolerance apparently requires (at present) no advisory statements on labels, likewise the presence of gluten as a causative factor in coeliac disease. The symptoms of these conditions closely overlap those of excessive consumption of low-digestible carbohydrates (Shaw et al. 1998), but only the latter require advisory statements on food labels. Risks associated with dietary fibre intake, such as impaired intake in the young or digestively compromised are given less prominence on food labels than are the risks of laxation. However, the differences in the way humans respond to dietary fibre polysaccharides on the one hand and LDCs such as sugar alcohols for example on the other is not absolute; they differ by degree. The development of LDCs of molecular weight intermediate between sugar alcohols and dietary fibre polysaccharides, for example polydextrose and fructooligosaccharides, bridges the distinctions that were once made. In addition, the properties of carbohydrates that cause them to be poorly digested and so contribute to abdominal discomfort are also responsible for their tooth friendly properties. Hence, risk and benefit are not always separable. Moreover, the risk of laxation caused by sugar alcohols may be perceived as a benefit among those vast numbers of people requiring laxatives. How food regulators view the low-digestible carbohydrates critically depends on the way the evidence has been conceived and the way it is presented. The present conference provides an opportunity to review the way we view low-digestible carbohydrates, how they might be considered in food regulations, and how relevant research can be better conducted.

\section{Tolerance and symptoms of intolerance: terminology}

Many different terms are used to describe the potential responses to LDCs (Table 3), and some are recognised symptoms of LDC consumption. For clarity the definitions of certain terms are given in the footnotes to Table 3. Some additional comment may be useful on the meaning of tolerance.

In lay language, tolerance means an ability to endure or willingness to allow. In the present context this could mean of one or more adverse symptoms. Few researchers have documented what people are willing to allow themselves to experience over the long term in the way of symptomatic responses to LDCs.

In medicine, tolerance is the development of unresponsiveness (e.g. to antibiotics) and intolerance is the development of responsiveness (e.g. glucose intolerance). Few studies have examined the development of responsiveness and unresponsiveness to LDCs, with the exception of their fermentation when another term is often used - adaptation.

With regard to LDCs, the usage of the term tolerance suggests it to mean a state in which there is an absence of unwanted symptoms of LDC consumption (Table 3). In particular, the absence from purgative actions and colic. In general the various responses mentioned in Table 3 result from the occurrence in the bowel of either too much water (leading for example to laxation and purgative action) or too much gas (leading for example to flatulence and colic).

\section{Laxation in context: nature's own}

Among the laxative foods are those that leave a large indigestible residue, such as cabbage, brown bread, oatmeal porridge and fruits containing rough seeds, low-digestible carbohydrates and vegetable acids (oxalic acid for example). Among these are honey (fructose), tamarinds, figs, prunes, raspberries, strawberries, stewed apples and senna. Foods that are very effective and may be defined as purgative because they cause watery stools associated with 
colic are aloes, rhubarb, cascara, senna and castor oil. Purgatives may be effective in removing irritants from the bowel that themselves cause diarrhoea or exacerbate undesirable systemic events such as hepatic coma. Lactulose and lactitol have found such usage (Heredia et al. 1988). In the context of food the distinction between purgative and laxative is seldom made. Thus usage in scientific and regulatory literature refers to laxative action rather than purgative action of LDCs, the latter being thought of as strong laxative action.

\section{Response to excess gas}

Because LDCs are poorly absorbed (Table 1) they enter the colon where they are subject to anaerobic fermentation. Gaseous endproducts are principally carbon dioxide, hydrogen and methane. The stoicheiometry of the fermentation process (Livesey \& Elia, 1996) indicates that $30 \mathrm{~g}$ of carbohydrate, an amount that may normally be fermented each day, releases gas at about $4 \mathrm{ml} / \mathrm{h}$ over $24 \mathrm{~h}$. Most people tolerate this rate of gas production. However, carbohydrates that both reach the colon rapidly and offer little resistance to fermentation may be used completely within about six hours (Thiébaud et al. 1984; Fritz et al. 1985). After a single oral dose of $30 \mathrm{~g}$ the rate of gas production is estimated stoicheometrically to average about $15 \mathrm{ml} / \mathrm{min}$. Interestingly, this rate of gas infusion at a steady rate into the human bowel is perceptible by the large majority of people (Serra et al. 1998). People experience one or a combination of symptoms described as pressure/ bloating, cramp/colic and sharp/stinging pain. Approximately $30 \%$ of people untrained to such rates of gas production suffer discomfort or pain. Whether people can learn (adapt) to tolerate such rates of gas production is not evident. Multiplying up by a factor of 3 (equal to the traditional number of meals per day) suggests an upper limit on grounds of gas production and abdominal discomfort of no more than $90 \mathrm{~g}$ fermentable LDC per day.

A significant find by Serra et al. (1998) was that people's semiquantitative response to intraluminal gas infusion was repeatable. They found gas perception, abdominal distension and gas retention in the colon were (a) related and (b) varied greatly between individuals. It appeared that intolerance of intraluminal gas was, in their study, associated with gas retention caused by low rates of evacuation. It has long been unknown whether differences in the tolerance of intraluminal gas are simply differences in perception to the same stimulus (gas load). The study by Serra et al. (1998) shows at least that differences in perception are related to differences in gas infusion and evacuation so that perception and load are indeed related.

Factors that alter the rate of gas production in vivo from LDCs are many. They include factors affecting gastric emptying (of which there are many), small intestinal transit (of which there are many modulators), fractional absorption of the LDC in the small intestine, delivery of water into the colon (causing mixing), which depends on the osmolality of the LDC (and its digestion products), and the susceptibility of the LDC to fermentation. Factors permitting tolerance include the extent of bowel fill during the time of fermentation, and ease of evacuation. Thus tolerance of
LDCs from the perspective of gas production is subject to many variables. From a regulatory standpoint all this can be simplified. All that is needed to trigger an advisory statement on a food label is knowledge about the circumstance in which the LDC is least tolerated. Whether excess water in the colon is tolerated less than excess gas is unclear. It seems likely that their effects would be broadly additive, water and gas together creating a volume of material that causes distension and requires to be evacuated as gas or eliminated as solids and liquid.

\section{Response to excess water}

Diarrhoea has various causes. Other than poisoning from micro-organisms and drugs, it results from starvation, food intolerance and malabsorption. Normally, the water lost in stools will average 40-60 g daily (Passmore et al. 1955) with a total stool weight of 100-120 g daily, although this may reach 250-350 g on any one day. In chronic diarrhoea stool weight may climb towards $2 \mathrm{~kg}$ and in acute diarrhoea it can be staggeringly higher, up to $20 \mathrm{~kg}$ due to cholera (Cummings, 1993). Consumption of indigestible sugars will elevate stool weight, largely due to water (and some biomass). Adults consuming $125 \mathrm{~g}$ lactulose daily, a disaccharide that is undigested in the upper gut, may lose $1.3 \mathrm{~kg}$ stool daily. However, $45 \mathrm{~g}$ lactulose (with three meals and a snack) causes an average stool output of about $300 \mathrm{~g}$, not much higher than the upper end of the normal output range (Hammer et al. 1989), but with the inconvenience of two toilet visits per day.

Stool weight after consumption of the indigestible and non-fermentable polyethylene glycol (PEG4000) is greater than after the consumption of an 'equiosmolar' amount of lactulose, suggesting that fermentation in the colon helps to prevent laxation by removal of the osmotic agent (Hammer et al. 1989). Adaptation to elevated rates of fermentation is facilitated by administration of progressively increasing doses so as not to wash the colon free of micro-organisms. In this way the tolerance of the low-digestible carbohydrates may be increased. Additionally, short-chain organic acids (SCOA) that are produced during fermentation encourage $\mathrm{Na}^{+}$and water absorption, and so promote tolerance. Such SCOAs fuel and maintain the colonic mucosa, and their absence during starvation is thought to be responsible, at least in part, for starvation diarrhoea. Also, hospitalised patients undergoing alimentation with simple, elemental nutrition without colonic substrates frequently have diarrhoea. Yet rates of fermentation in excess of habitual rates will also cause loose stools, such as after intracolonic starch (Hammer et al. 1989). Thus diarrhoea is a result of both too little and too much colonic substrate.

A variety of factors associated with the fermentation process may affect wateriness of the stool. Rapid fermentation of LDC, such as after a large increase in the dose administered, may yield lactate and short-chain organic acids at a faster rate than can be absorbed so temporarily promoting diarrhoea. Unfermented polysaccharides may also bind water while fermentable polysaccharides, oligosaccharides, sugar and sugar alcohols generate biomass during fermentation, which also has water-holding capacity and so helps to reduce the 


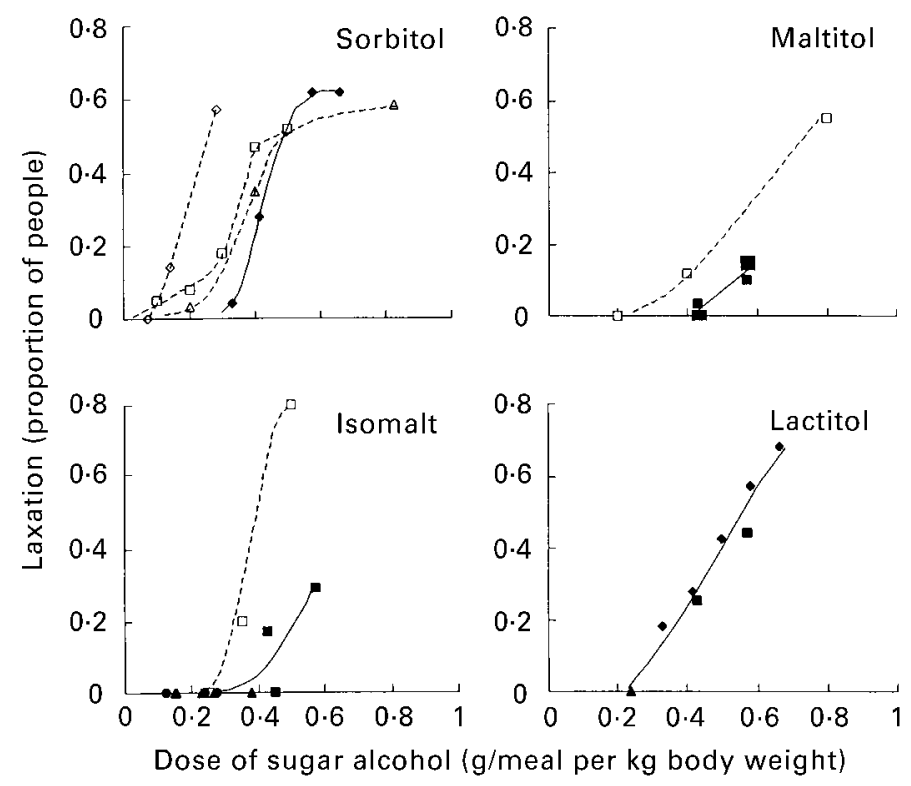

Fig. 1. Laxation-dose curves for sorbitol and disaccharide alcohols in adults of mixed gender (and children taking isomalt). 'Meals' were either single drinks or jelly (broken curves) or solid foods (continuous curves). Solid foods were either a single food (chocolate) or multiple meals (with intakes average per meal). Sorbitol: adults taking either drinks $(\Delta$; Koizumi et al. 1983; $\diamond$; Hyams, 1983) or jelly ( $\square$; Oku \& Okazaki, 1996) or multiple meals with a short period for adaptation ( $\bullet$; Patil et al. 1987). Maltitol: adults taking either a drink ( $\square$; Koizumi et al. 1983) or chocolate (ロ; Storey et al. 1997; -; Koutsou et al. 1996). Isomalt: adults taking drinks ( $\square$; Pütter \& Spengler, 1975 as note in JECFA, 1987) or chocolate (ם; Lee et al. 1994; Koutsou et al. 1996) or multiple meals after adaptation ( $\mathbf{\Lambda}$; Fritz et al. 1985; Spengler et al. $1987)$ and children (<12 y) taking multiple meals after adaptation (@; Paige et al. 1992). Lactitol: adults taking chocolate (ם; Koutsou et al. 1996), multiple meals ( $\bullet$; Patil et al. 1987) and multiple meals after adaptation ( $\mathbf{\Delta}$; van Es et al. 1986). Data points are means for groups of between 10 and 83 people. For calculation of the present data, a body weight of $70 \mathrm{~kg}$ (Western subjects) was assumed when a specific value was needed but not reported in the primary literature. Laxation was the increment in number or people with loose faeces above basal or control (often sucrose) as a proportion of the number of people investigated.

wateriness of the stool. The water binding by unfermented carbohydrate and biomass also counteract the production of the hard stools associated with constipation and so facilitates laxation.

Water is actively secreted into the upper gastrointestinal tract after a meal. Additional water may be drawn into the small intestine to maintain osmolality when certain LDCs are present. An absence of absorbable nutrients reduces water absorption associated with nutrient absorption. These processes eventually lead to an elevated water load entering the colon when osmotically active LDCs are ingested. The capacity of the human colon for water absorption is 6-8 litres daily (Debongnie \& Phillips, 1978). By slowing gastric emptying absorbable nutrients lower the rate at which an osmotic load can enter the small intestine and so lowers the water load to the colon (Livesey, 1990). Osmotically active LDCs are more readily tolerated therefore when taken with a meal.

\section{Product acceptability, not tolerance of the LDC may sometimes limit symptoms of LDC consumption}

Many of the symptoms (Table 3) experienced after LDC consumption, although thought of as undesirable, may have no impact on acceptability of the products containing LDC. This seems surprising at first, but the answer lies in the acceptability of the food product as much as the acceptability of the LDC. For example, the usual consumption of chocolate may be limited on average to approximately $60 \mathrm{~g}$ per day in young adults and $35 \mathrm{~g}$ per day in adults over 43 years of age. With these intakes, chocolate based on isomalt has too little of this polyol (16 g at $60 \mathrm{~g}$ chocolate) to cause symptoms that deter consumption. Thus free consumption of isomalt- and sucrose-based chocolate are identical (Zumbé \& Brinkworth, 1992). However, per unit mass sorbitol is more osmotic than isomalt, and will cause consumers to reduce their chocolate consumption when replacing either sucrose or isomalt (Zumbé \& Brinkworth, 1992). For this reason sorbitol is being replaced in confectionery by more tolerable LDCs.

\section{Laxation with sorbitol: a case study interrelating experimental observations and food regulations}

Sorbitol was introduced as a therapeutic under the name 'Sionin' (Thannhauser \& Meyer, 1929). Early concerns 
about sorbitol-induced laxation arose with dietetic candy (Gryboski, 1966) and later in paediatric medicine where sorbitol was used as a sweet base for infant and children's medicines (Charney \& Bodurtha, 1981; Hyams, 1982, 1983). In food studies much of what has been learned is based on experiments in adults.

Fig. 1 collects together observations from several studies on laxation with sorbitol. Gender may affect laxation when expressed per $\mathrm{kg}$ body weight (see below), it should therefore be noted that the curves in Fig. 1 are for mixed gender. Laxation is clearly less when sorbitol is administered on a full stomach (solid curves). It appears similar after incorporation into a drink and a nutrient free jelly, when each is taken on an empty stomach. However, there are no properly conducted interlaboratory trials to establish reliability of trigger levels causing laxation. In the absence of such trials, doses of LDCs that trigger laxation as given in food regulations will inevitably be thought of with uncertain reliability.

There is evidence of similar results for sorbitol when presented in similar forms in different laboratories (see Koizumi et al. 1983), although data published at about the same time by Hyams (1983) suggest differently. Differences in ethnicity of the subjects appears not to be a major cause of variation in results for sorbitol for which tolerance appears to be similar in Westerners, Japanese, Blacks and Asians (Koizumi et al. 1983; Jain et al. 1987). Thus it seems possible to have relevant food regulations that are broadly applicable across national and ethnic boundaries.

The laxation curves (Fig. 1) show certain features. Firstly, they are essentially sigmoidal and an $\mathrm{ED}_{50}$ can be determined to describe when $50 \%$ of the population of subjects develop diarrhoea (when defecation may then be twice or more per day). Although an $\mathrm{ED}_{50}$ may be useful descriptively, to date it has no application in food regulations. A second feature is the maximum no-effect dose or laxative threshold. This has applications in food regulations and provides a basis on which to identify a dose of LDC in a food product that might trigger a requirement to issue an advisory statement about the possibility of laxation on the food label.

The maximum no-effect dose differs between individuals; for some people it is quite high. It is this variation between people that gives the shape to the curves in Fig. 1. Thus some people tolerate quite high doses of sorbitol before reaching their individual maximum no-effect dose. For regulatory purposes it is the sample population's maximum no-effect dose that is relevant, that is this dose in the more sensitive people. Two difficulties arise: with sigmoidal curves a maximum no-effect dose may not be clearly identifiable (curve tends to meet the $x$-axis as a tangent). Second, how large a sample of the population is needed to estimate the population maximum no-effect dose?

Laxation has a number of ill-defined determinants. The range of doses over which each curve in Fig. 1 rises is relatively wide, indicating considerable interindividual differences, with some people being fairly resistant to laxation. This feature is evident in both males and females. Very noticeable, however, is a gender difference in sorbitol tolerance with regard to laxation, when expressed per $\mathrm{kg}$ body weight! Females then appear more tolerant than males, an observation that is repeatable and not restricted to sorbitol (Koizumi et al. 1983; Oku \& Okazaki, 1996). Whether the difference is innate or secondary to other factors or is entirely due to the body weight difference between genders is unclear. There is evidence of a higher degree of constipation among women than men (Koizumi et al. 1983) and laxatives are less effective in constipated people (Tramonte et al. 1997). Also, stomach emptying may be slower in women. With regard to food regulations any gender difference is not critical; in practice the maximum no-effect dose for the population will be independent of possible differences between genders provided both sexes are adequately represented in the sampled population.

It is well known that laxation occurs more readily when low-molecular-weight LDCs are consumed in drinks than with meals. Europe has proposed that such carbohydrates should not be used as sweeteners in drinks (European Council Directive, 1990). This means there is little value in determining trigger levels for the purpose of food regulations based on studies where carbohydrate is administered as a drink on an empty stomach. Nevertheless, for experimental purposes the administration of osmotically active LDCs in drink form appears to be acceptable after a meal, and so the directive may be too restrictive. However, with such a directive it may be argued that studies on tolerance should focus on feeding with a meal (although see candies below). What comprises a meal is unclear. Prior ingestion of food appears to result in a rightward shift in the laxation curve, with the $\mathrm{ED}_{50}$ increasing nearly twofold. Candies may contain sorbitol (or other LDCs) that could be consumed on an empty stomach. The quantitative impact on laxation due to the simultaneous absence of both water (drink) and macronutrients is unclear, although results may soon be available (A Lee, personal communication). A decrease in the water content of a drink improves the intestinal tolerance of fructose (Ravich et al. 1983). Without such knowledge of LDCs it may be presumed that trigger levels in food regulations could be set cautiously low.

\section{Adaptation}

A feature of the laxation studies on polyols, is that they have been undertaken in subjects variably adapted to them. Adaptation is likely to have at least two consequences on laxation.

Firstly, water retention may improve in both the small and large intestine. A capacity for adaptation in the small intestine is evident from a comparison of the passage of ileal fluid in intact and colectomised subjects (having ileostomies). Ileal fluid flow in intact adults averages a rate of 2 litres per day (Debongnie \& Phillips, 1978), whereas in ileostomates it is reduced threefold to about $600 \mathrm{ml}$ daily (Livesey et al. 1995). This suggests an adaptation in the balance of fluid secretion and absorption may occur at the level of the upper gut, although in part, this may be due to restricted fluid intake in ileostomates.

Second, large bowel microbial ecology and enzyme induction favours a more proximal and, at high doses, more 
complete fermentation. The greater the proportion of the LDC that is fermented and absorbed, the lower will be the osmotic activity. The more proximally the fermentation occurs, the more time there will be for water absorption in the large bowel.

Third, in the laboratory, laxation is a placebo response, which wears off with time (weeks) (Marteau \& Flourié, this issue). Possibly, laboratory based studies will overexaggerate the laxative effects of low-digestible carbohydrates. Moreover, adaptative laxation during weeks of LDC consumption may in part be due to placebo effects wearing off.

The shape of the curves in Fig. 1 will depend in part on how quickly adaptation takes place when curves are obtained by serial incremental dosage. Trigger levels for regulatory purpose may need to be recognised as levels in unadapted people (despite the possible placebo effect), with a recognition that people may habituate to consume higher doses without ill-effect.

\section{Lessons from erythritol and tagatose}

Erythritol and D-tagatose are carbohydrates recently proposed for human consumption. Neither is digested in the sense of enzymatic cleavage since both are carbohydrate monomers. Also erythritol is substantially absorbed. Being a monosaccharide derivative, erythritol is not a sugar but a sugar alcohol (a tetrose alcohol, $\mathrm{C}_{4} \mathrm{H}_{10} \mathrm{O}_{4}$ ). It is derived from sucrose or $\mathrm{D}$-glucose of wheat and maize by fermentation with the Aureobasidium sp. yeast. D-Tagatose is a sugar, and may be produced commercially from the keto-analogue of milk galactose. D-Tagatose differs from D-fructose by rotation at carbon 4 . Almost as much tagatose is metabolised as is absorbed, whereas absorbed erythritol is largely excreted in urine. These materials find a place among the low-digestible carbohydrates because the symptoms of excess consumption are broadly the same as those experienced with other LDCs (Oku \& Okazaki, 1996; Buemann et al. 1999a,b), because erythritol is a sugar alcohol and because tagatose is poorly absorbed (Johansen \& Jensen, 1997).

As expected from its molecular structure (Livesey, 1992), erythritol is extensively but not completely absorbed by the human small intestine (Oku \& Noda, 1990). Absorption occurs by passive diffusion and so the loss to the colon will depend, like sorbitol, on the amount ingested when taken with water on an empty stomach. Observations by Oku \& Okazaki (1996) show that when taken in jelly, erythritol is approximately four times less laxative than sorbitol. As with sorbitol, there are possible gender differences. Such data provide firm evidence that the laxative threshold varies with the source of polyol, and provides scope for regulatory authorities to aim at tabulation of the various trigger levels for individual polyols should this be helpful. Although erythritol is less laxative than sorbitol, the latter induces less nausea (Oku \& Okazaki, 1996), presumably because of the difference in osmotic activity per gram. Abdominal discomfort with erythritol is associated with gas production and laxation rather than nausea.

Studies by Lee \& Storey (1999) with $20 \mathrm{~g}$ tagatose or lactitol in $50 \mathrm{~g}$ chocolate yielded a higher incidence of nausea among 50 subjects for tagatose while both treatment groups endured quantitatively similar symptoms arising from the large bowel. Again, this is consistent with nausea being due to upper gastrointestinal water or osmotic activity, and unconnected to large bowel events.

Studies with erythritol and tagatose (above) show them to be weak emetics and this is presumed to be due to their high osmolarity. However, on this ground alone there is no reason why tagatose should be more emetic than sorbitol or other monosaccharide alcohol, e.g. mannitol. At the time of writing such comparative data are absent from the literature.

\section{Gender}

Gender difference in laxation with sorbitol and erythritol is a possibility as noted above. Interestingly, nausea after erythritol has been observed in women only. Similarly, after tagatose nausea only occurred in women. This led Buemann et al. (1999a) to speculate that women are more susceptible to nausea than men, although men are not entirely resistant (Buemann et al. 1999b). Potentially, the LDCs are retained in the upper gastrointestinal tract more readily in women than in men causing greater nausea and less laxation in women. This may be related to differences in stomach emptying among men and women.

For erythritol at least, it needs to be considered whether the laxative threshold may not be an adequate end-point for regulatory purposes, rather nausea and emesis among consumers could be more problematic. Emesis has approximately half the prevalence of reported nausea after erythritol.

\section{Age}

The effect of age on tolerance is unclear. Tolerance studies are sometimes undertaken with young adults, for example age 40 or less (Buemann et al. 1999a,b; Lee \& Storey 1999). These people may be less tolerant than people $>40$ years old just as they are less susceptible to constipation. Alternatively, they could be more tolerant than people $>40$ years old because of their relative youth. Children 8-12 years old appear to be as tolerant as children $>12$ years old with respect to laxation and other symptoms when administered with the mixed disaccharide alcohol isomalt up to $35 \mathrm{~g}$ in a single dose in chocolate (Paige et al. 1992). This corresponds to an intake of up to $0.85 \mathrm{~g} / \mathrm{kg}$ body weight per $d$ in the younger group of children compared with approximately $0.5 \mathrm{~g}$ isomalt $/ \mathrm{kg}$ body weight established as a tolerable dose per eating occasion in adults (Fritz et al. 1985). This difference is quite large and may indicate that the expression 'per $\mathrm{kg}$ body weight' gives a false impression of tolerance in children compared to adults. Although these studies are difficult to compare it remains possible that children may be more tolerant of isomalt on a body weight basis than adults. Adults and children $(>8$ years old) show similar tolerance of isomalt in candies when expressed on an intake per day basis (Lee, personal communication), implying greater tolerance on a per kg body weight basis in children. The roles of other 
Table 4. Estimates of the laxative thresholds (maximum no-effect dose) for selected low-digestible carbohydrates

\begin{tabular}{|c|c|c|c|c|c|c|}
\hline & \multirow{2}{*}{$\frac{\text { In drinks and jelly }}{\text { (g/meal per } \mathrm{kg})}$} & \multirow{2}{*}{$\frac{\text { Increment with food }}{(\mathrm{g} / \text { meal per } \mathrm{kg})}$} & \multicolumn{4}{|c|}{ In foods } \\
\hline & & & (g/meal per $\mathrm{kg}$ ) & (g/product in adults) & (g per $100 \mathrm{~g}$ of product) & (g/day) \\
\hline Erythritol & 0.66 & - & $>0.66^{a}$ & $>46^{\mathrm{a}}$ & $>66^{a}$ & $>132^{a}$ \\
\hline Sorbitol & $<0.21^{\mathrm{b}}$ & $0 \cdot 11$ & $\begin{array}{l}<0.30^{\mathrm{b}} \\
<0.11^{\mathrm{b}}\end{array}$ & $\begin{array}{r}<22^{b} \\
<8^{b}\end{array}$ & $\begin{array}{l}<32^{\mathrm{b}} \\
<11^{\mathrm{b}}\end{array}$ & $\begin{array}{l}<66^{b} \\
<24^{b}\end{array}$ \\
\hline Maltitol & 0.29 & 0.13 & 0.42 & 29 & 42 & 87 \\
\hline Isomalt & 0.25 & 0.07 & 0.32 & 22 & 32 & 66 \\
\hline Lactitol & - & - & 0.25 & 18 & 25 & 54 \\
\hline Polydextrose & - & - & $0.46^{\mathrm{c}}$ & $32^{c}$ & $46^{\mathrm{C}}$ & $96^{c}$ \\
\hline Column number ${ }^{d}$ & 1 & 2 & 3 & 4 & 5 & 6 \\
\hline
\end{tabular}

a Based on the assumption that it will be greater after meals than after jellies.

${ }^{b}$ Values for sorbitol are given as an upper limit because its threshold is unclear. Data from Zumbé \& Brinkworth (1992) indicate a lower value is probable under certain circumstances. Variability for sorbitol perhaps reflects its variable absorption.

${ }^{c}$ Data are based on a $1 \mathrm{~g} / \mathrm{kg}$ intake in children assuming three meals per day.

${ }^{d}$ Data in columns 1 and 3 are either from Fig. 1, or for erythritol from Oku and Okazaki (1996) and polydextrose from Murray (1988). For sorbitol additional data are from Zumbé \& Brinkworth (1992). Data in column 2 are differences between columns 1 and 3 . Data in column 4 are those in column 3 multiplied by 70 , the average weight of a western adult. Data in column 5 are those in column 4 multiplied by 100 and divided by 70 , representative of a $70 \mathrm{~g}$ food product. Data in column 6 are those in column 3 multiplied by 3 (representing three evenly spread meals throughout the day in amounts sufficient to maintain energy balance).

factors in this comparison await publication of the full results. Children and possibly adults readily accommodate up to $1 \mathrm{~g} / \mathrm{kg}$ body weight polydextrose daily (Murray, 1988). Again, however, the data in the two age groups are not strictly comparable.

\section{Infants and preschool children}

Diarrhoea is up to six time more common in this age group than in adults (Payne et al. 1997). Undeveloped gastrointestinal capacity is likely to be responsible. In this group it is well known that fruit juice may exacerbate the problem. It seems probable that three-year olds are at greatest risk of diarrhoea from sorbitol consumption. All children in this age group may have afebrile diarrhoea in response to intakes of $0.5 \mathrm{~g}$ sorbitol $/ \mathrm{kg}$ body weight per day (or less than a day), compared to $50-60 \%$ of adults consuming the same dose in one meal (Fig. 1). In children of a younger age than 3 years, access to sorbitol-containing foodstuffs is possibly limited except through medication. However, vitamin $\mathrm{C}$ supplementation of infants is common, and products including sorbitol are not advised (Hill \& Kamath, 1982). The occurrence of sorbitol-induced diarrhoea is difficult to find in 4- and 5-year olds (Payne et al. 1997).

\section{Ingestion frequency: expression of dose}

The daily dose of LDC that may be ingested before breaching the laxative threshold depends on the frequency of its ingestion. Ingestion of a given dose during two or three occasions breaches the threshold less readily than a single dose of the same total amount, as indicated for erythritol and sorbitol (see Oku \& Okazaki, 1996) and as evident for lactitol and isomalt from the studies by van Es et al. (1986) and Spengler et al. (1987), respectively. This is because laxation depends on the rate of entry of osmotically active agent into the small intestine. This rate is obviously decreased when ingestion is spread across the day. Moreover, co-ingestion of food slows gastric emptying. Care has to be taken, therefore, over the meaning of dose.

The food labelling regulation provided by Codex (1991) states: 'If the food provides a daily intake of sugar alcohols in excess of $20 \mathrm{~g}$ per day, there shall be a statement on the label to the effect that the food may have a laxative effect'. Such a provision can lead to false expectations. It is possible to consume two or more items of food on one occasion that would breach this laxative threshold, but without warning of laxation on the label. Moreover, it is possible to consume more than $20 \mathrm{~g}$ per day without experiencing the laxative effect suggested on the label when the intake is spread throughout the day.

Proposals by the Australian and New Zealand Food Authority (ANZFA, 1999) differ from the Codex regulation. Cognisant that the co-ingestion of food affects the laxative effect, and that an expression is needed to be applied to individual food items, they proposed that foods shall carry an advisory statement to the effect that 'a food may be laxative when its content exceeds a threshold of $x \mathrm{~g}$ per $100 \mathrm{~g}$ food', the value of $x$ depending on the sugar alcohol. Such a provision would cause certain food items of low serving portion (by weight) to require a laxative statement even though they would be far from laxative.

Among the regulatory and scientific literature, dose is expressed in units of weight (Lee et al. 1994; Storey et al. 1998; Buemann et al. 1999a,b), weight per day (Patil et al. 1987; Codex, 1991), weight per unit weight of food (ANZFA, 1999), and weight per unit body weight (Murray, 1988; Oku \& Okazaki, 1996; Paige et al. 1992). Other more useful units are possible. Thus, the key determinant is the dose at each meal (since upper gastrointestinal effects take place meal-by-meal). Observations can be expressed for all studies in units of grams ingested per meal (or product) or $g$ ingested per meal (product) per kg body weight with mostly good agreement between the studies of various designs (Fig. 1). Such expression yields useful information in terms of the food item (Table 4).

The approximate laxative thresholds or maximum noeffect doses are given in Table 4 for selected sugar alcohols. The data have been expressed in several ways. For all the LDCs tabulated, except sorbitol, products may be formulated with either at or in excess of $25 \mathrm{~g} / 100 \mathrm{~g}$ of food. Such products consumed at up to $70 \mathrm{~g}$ per occasion (a regular weight of confection at the present time) would not normally cause loose stools. 
Further, up to three such $70 \mathrm{~g}$ products could be eaten per day by the reference weight adult $(70 \mathrm{~kg})$ provided they were evenly spread across the day. It should be noted that the data in Table 4 were computed with zero margin of 'safety'. A natural margin is set by people not consuming such confections alone.

Data may be examined across the LDC products. Erythritol is well tolerated because it is substantially absorbed. Sorbitol is partly absorbed but of lower molecular weight than maltitol consistent with it being less well tolerated. With sorbitol a clear threshold is more difficult to find than with other polyols and diarrhoea may be found in people consuming $8 \mathrm{~g}$ sorbitol in chocolate even though none is experienced by the same people consuming $16 \mathrm{~g}$ isomalt (Zumbé \& Brinkworth, 1992). For this reason it is possible at this time to place only an upper limit on the tolerance data for sorbitol (Table 4). Variable outcomes for sorbitol probably relate to variable extents of digestion and absorption according to circumstance. The tolerance of the disaccharide alcohols maltitol, isomalt and lactitol broadly ranks with their relative digestibilities. Based on children consuming polydextrose, this LDC is possibly less well tolerated than one would predict on the basis of its molecular weight. Presumably this is because the product is partially fermented and so the polydextrose and its digestion products continue to express osmotic activity in the colon. Potentially also, the fermentation may occur more distally giving less time for water absorption.

\section{Food labelling regulations}

Foodstuffs containing low-digestible carbohydrates may be required to give an advisory statement to say that they may have a laxative effect. Critical information that could trigger the requirement of such a statement, should one be needed, is such as given in column 4 of Table 4; that is, as an amount per product (of serving size) per subject. On this basis, there is at this time no observation or reason for a threshold to be set at different levels for adults and children ( $>8$ years of age), nor a sound basis for distinguishing between genders. There is also no observational basis for selecting to express a threshold as a percentage of a food product. Reasonable values based on Table 4 column 4 would be $10 \mathrm{~g}$ per product (of serving size) for monosaccharide hexitols, $20 \mathrm{~g}$ for disaccharide hexitols and perhaps $30 \mathrm{~g}$ for oligosaccharides or oligosaccharide hexitols. There are however, always those who will be sensitive to low-digestible carbohydrates, whatever they are and in whatever form they are consumed. These people are perhaps the ones who would be guarded most by a warning label. However, it is unclear whether the different thresholds for the various low-digestible carbohydrates would be meaningful for them. It may also be regarded that the range of thresholds between individual consumers is broader than the range of threshold for individual low-digestible carbohydrates (Fig. 1), in which case setting different thresholds for each low-digestible carbohydrate may be meaningless for each of a majority of consumers. On the other hand, the low threshold for sorbitol has now resulted in manufacturers preferring matitol, isomalt or lactitol without detailed labelling regulations, suggesting that selfregulation has been effective.

\section{References}

ANZFA (1999) Review of the Provisions for Low-joule Foods and Carbohydrate Modified Foods. Canberra: Australia New Zealand Food Authority.

Bornet FRJ, Blayo A, Dauchy F \& Slama G (1996) Gastrointestinal response and plasma and urine determinations in human subjects given erythritol. Regulatory Toxicology and Pharmacology 24, S296-S302.

British Nutrition Foundation (1990) Complex Carbohydrates in Foods. The Report of the British Nutrition Foundation's Task Force on Complex Carbohydrates. London: Chapman and Hall.

Buemann B, Toubro S, Raben A \& Astrup A (1999a) Human tolerance to a single, high dose of tagatose. Regulatory Toxicology and Pharmacology 29, S66-S70.

Buemann B, Toubro S \& Astrup A (1999b) Human gastrointestinal tolerance to D-tagatose. Regulatory Toxicology and Pharmacology 29, S71-S77.

Canada (1993) Amendment. Food and Drug Regulations SOR/93276 2601-2808. Montreal: Health Canada.

Charney EB \& Bodurtha JN (1981) Intractable diarrhoea associated with the use of sorbitol. Journal of Paediatrics $\mathbf{9 8 ,}$ $157-158$.

Codex (1991) Codex standard for formula foods for use in weight control diets. Codex Standard 181.

Collins MD \& Gibson GR (1999) Prebiotics, probiotics and synbiotics: approaches for modulating the microbial ecology of the gut. American Journal of Clinical Nutrition 69, S1052S1057.

Cummings JH (1993) Nutritional management of diseases of the stomach and bowel. In Human Nutrition and Dietetics, pp. 480-506 [JS Garrow and WPT James, editors]. London: Churchill Livingstone.

Cummings JH, Bingham S, Heaton KW \& Eastwood MA (1992) Faecal weight, colon cancer risk and dietary intake of nonstarch polysacchrides (dietary fibre). Gastroenterology 103, 1783-1789.

Cummings JH \& MacFarlane GT (1999) Probiotics and prebiotics: can regulate the activities of the intestinal bacteria, benefits health. British Medical Journal 318, 999-1003.

Debongnie JC \& Phillips SF (1978) Capacity of the human colon to absorb fluid. Gastroenterology 74, 698-703.

Ellis FW \& Krantz JC (1941) Sugar alcohols XXIV. The metabolism of sorbitol in diabetes. Annals of Internal Medicine 18, 792-796.

European Council Directive (1990) Proposal for a council directive on sweeteners for use in foodstuffs. Official Journal of the European Community 4, (C242).

FDA (1998) Polydexrose. Code of Federal Regulations 21 Ch.184-85. Washington, DC: Food and Drug Administration.

Fritz M, Siebert G \& Kasper H (1985) Dose dependency of breath hydrogen and methane in healthy volunteers after ingestion of a commercial disaccharide mixture, Palatinit. British Journal of Nutrition 54, 389-400.

Gavin J, Ellis T, Dewar AL, Rolles CJ \& Connett GT (1997) Dietary fibre and the occurrence of gut symptoms in cystic fibrosis. Archives of Digestive Diseases in Childhood 76, 3537.

Gryboski JD (1966) Diarrhea from dietetic candy. New England Journal of Medicine 275, 718.

Hammer HF, Santa Ana CA, Schiller LR \& Fordtran S (1989) Studies on osmotic diarrhea induced in normal subjects by 
ingestion of polyethylene glycol and lactulose. Journal of Clinical Investigation 84, 1056-1062.

Heredia D, Terés J, Orteu N \& Rodés J (1988) Lactitol vs. lactulose in the treatment of chronic recurrent portal-systemic encephalopathy. Journal of Hepatology 7, 106-110.

Hill RE \& Kamath KR (1982) 'Pink' diarrhoea: osmotic diarrhoea from a sorbitol-containing vitamin $\mathrm{C}$ supplement. Medical Journal of Australia 1, 387-389.

Hyams JS (1982) Chronic abdominal pain caused by sorbitol malabsorption. Journal of Pediatrics 100, 772-773.

Hyams JS (1983) Sorbitol intolerance: an unappreciated cause of functional gastrointestinal complaint. Gastroenterology 84, 3033.

Jain NK, Patel VP \& Pitchumoni CS (1987) Sorbitol intolerance in adults: prevalence and pathogenesis on two continents. Journal of Clinical Gastroenterology 9, 317-319.

JECFA (1987) Toxicological Evaluation of Certain Food Additives and Contaminants. Series number 20. Geneva: FAO/WHO.

Jenkins DJA \& Kendall CWC (1998) Dietary fibre, the evolution of the human diet and coronary heart disease. Nutrition Research 18, 633-652.

Jenkins DJA, Wolever TM, Taylor RH, Barker H, Fielden H, Baldwin JM, Bowling AC, Newman HC, Jenkins AL \& Goff DV (1981) Glycemic index of foods: a physiological basis for carbohydrate exchange. American Journal of Clinical Nutrition 34, 362-366.

Johansen HN \& Jensen BB (1997) Recovery of energy as SCFA after microbial fermentation of D-tagatose. International Journal of Obesity Suppl 121, S50.

Kandelman D (1997) Sugar, alternative sweeteners and meal frequency is related to caries prevention: new perspectives. British Journal of Nutrition 77, S121-S128.

Koizumi N, Fujii M, Ninomiya R, Inoue $Y$, Kagawa $T$ \& Tsukamoto $T$ (1983) Studies on transitory laxative effects of sorbitol and maltitol I: Estimation of $50 \%$ effective dose and maximum non-effective dose. Chemosphere 12, 45-53.

Koutsou GA, Storey DM, Lee A, Zumbé A, Flourie B, Le Bot Y \& Oliver PH (1996) Dose related gastrointestinal response to the ingestion of either isomalt, lactation or maltitol in milk chocolate. European Journal of Clinical Nutrition 50, 17-21.

Lederle FA, Busch DL, Mattox KM, West MJ \& Aske DM (1990) Cost-effective treatment of constipation in the elderly: a randomized double blind comparison of sorbitol and lactulose. American Journal of Medicine 89, 597-601.

Lee A \& Storey DM (1999) Comparative gastrointestinal tolerance of sucrose and lactitol, or D-tagatose in chocolate. Regulatory Toxicology and Pharmacology 29, S78-S82.

Lee A, Zumbé A \& Storey D (1994) Breath hydrogen after ingestion of the bulk sweeteners sorbitol, isomalt and sucrose in chocolate. British Journal of Nutrition 74, 731-737.

Livesey G (1990) The impact of the concentration and dose of Palatinit ${ }^{\mathrm{R}}$ in foods and diets on energy value. Food Sciences and Nutrition 42F, 223-243.

Livesey G (1992) Energy values of dietary fibre and sugar alcohols for man. Nutrition Research Reviews 5, 61-84.

Livesey G \& Elia M (1996) Short chain fatty acids as an energy source in the colon: metabolism and clinical implications. In Physiological and Clinical Aspects of Short Chain Fatty Acids, pp. 427-481 [JH Cummings, JL Rombeau and T Sakata, editors]. Cambridge: Cambridge University Press.

Livesey G, Wilkinson JA, Roe MA, Faulks RM, Clark S, Brown JC, Kennedy H \& Elia M (1995) Influence of the physical form of barley grain on the digestion of its starch in the human small intestine and implications for health. American Journal of Clinical Nutrition 61, 75-81.

Miller JCB \& Lobbezoo I (1994) Replacement of starch with sucrose in a high glycaemic index breakfast cereal lowers glycaemic and insulin responses. European Journal of Clinical Nutrition 48, 749-752.

Moynihan PJ (1998) Update on the nomenclature of carbohydrates and their dental effects. Journal of Dentistry 26, 209-218.

Murray PR (1988) Polydextrose. In Low-Calorie Products, pp. 83-100. London: Elsevier Applied Science.

Oku T \& Noda K (1990) Erythritol balance study and estimation of the metabolizable energy of erythritol. In Caloric Evaluation of Carbohydrates, pp. 65-75 [N Hosoya, editor]. Tokyo: Research Foundation for Sugar Metabolism.

Oku T \& Okazaki M (1996) Laxative threshold of sugar alcohol erythritol in human subjects. Nutrition Research 16, 577-589.

Paige DM, Baless TH \& Davis LR (1992) Palatinit digestibility in children. Nutrition Research 12, 27-37.

Passmore R, Meiklejohn AP, Dewar AD \& Throw RK (1955) Energy utilization in overfed thin young men. British Journal of Nutrition 9, 20-27.

Patil DH, Grimble GK \& Silk DAB (1987) Lactitol, a new hydrogenated lactose derivative: intestinal absorption and laxative threshold in normal human subjects. British Journal of Nutrition 57, 195-199.

Payne ML, Craig WJ \& Williams AC (1997) Sorbitol is a possible risk factor for diarrrhea in young children. Journal of the American Dietetic Association 97, 532-534.

Ravich WJ, Bayless TM \& Thomas M (1983) Fructose: incomplete intestinal absorption in humans. Gastroenterology 84, 26-29.

Serra J, Azpiroz F \& Malagelada J-R (1998) Intestinal gas dynamics in humans. Gastroenterology 115, 542-550.

Shaw JS, Brooks JL, Dickerson JWT \& Davies GJ (1998) Dietary triggers in irritable bowel syndrome. Nutrition Research Reviews 11, 279-309.

Spengler M, Somogyi JC, Pletcher E \& Boehme K (1987) Tolerability, acceptance and energetic conversion of isomalt (Palatinit) in comparison with sucrose. Aktulle Ernährungsmedizin 12, 210-214.

Storey DM, Koutsou G, Lee A, Zumbé A, Olivier P, Le Bot Y \& Flourié B (1998) Tolerance and breath hydrogen excretion following ingestion of maltitol incorporated at two levels into milk chocolate consumed by healthy young adults with and without fasting. Journal of Nutrition 128, 587-592.

Thannhauser SJ \& Meyer KH (1929) Sorbit (Sionin) als Kohlehydraterstz für den Diabeteskranken. Müchener Medizin Wochenschrift 76, 356-366.

Thiébaud D, Jacot H, Schmitz M, Spengler M \& Felber JP (1984) Comparative study of isomalt and sucrose by means of continuous indirect calorimetry. Metabolism 33, 808-812.

Tramonte SM, Brand MB, Mulrow CD, Amato MG, O'Keefe ME \& Ramirez G (1997) The treatment of chronic constipation in adults - a systematic review. Journal of General Internal Medicine 12, 15-24.

Van Es AJH, De Groot L \& Vogt JE (1986) Energy balance of eight volunteers fed on diets supplemented with either lactitol or saccharose. British Journal of Nutrition 56, 545-560.

Zumbé A \& Brinkworth RA (1992) Comparative studies of gastrointestinal tolerance and acceptability of milk chocolate containing either sucrose, isomalt or sorbitol in healthy consumers and type II diabetics. Zeitschrift für Ernährungswissenschaft 31, 40-48. 